\title{
Gastrointestinal bleeding in pregnant patients with cirrhosis: Pathophysiology, review of outcomes, and management
}

\author{
Abdussalam Shredi MD, Benjamin Elberson PhD
}

\begin{abstract}
Pregnancy is a rare event in patients with cirrhosis because the metabolic and hormonal changes associated with cirrhosis lead to anovulation and amenorrhea. Liver cirrhosis with portal hypertension is considered one of the most common causes of gastrointestinal bleeding in general and comes after peptic ulcer disease. Gastrointestinal bleeding in the setting of portal hypertension occurs secondary to either esophageal varices or portal hypertensive gastropathy. Its management can be challenging in general, and pregnancy can only make it more complicated. In this article we will review the management of variceal gastrointestinal bleeding in pregnancy.
\end{abstract}

Keywords: gastrointestinal bleeding, pregnancy, cirrhosis, portal hypertension, varices, esophagogastroduodenoscopy

\section{INTRODUCTION}

Cirrhosis complicating pregnancy is not common, but when it occurs, it carries a maternal and fetal mortality rate as high as $50 \% .^{1}$ Pregnant patients with cirrhosis face unique risks, including higher rates of spontaneous abortion, prematurity, life-threatening variceal hemorrhage, hepatic decompensation, splenic artery aneurysm rupture, and postpartum hemorrhage. ${ }^{1}$ Bleeding from esophageal varices can occur in $60-80 \%$ of pregnant patients with cirrhosis, ${ }^{2}$ because the physiologic changes in pregnancy worsen portal hypertension. It is therefore crucial for physicians caring for pregnant patients with cirrhosis to understand how to approach potential complications and provide necessary treatment.

Corresponding author: Abdussalam Shredi Contact Information: Abdussalam.shredi@ttuhsc.edu DOI: 10.12746/swrccc.v6i25.479

\section{Changes to hepatic and hematologic PHYSIOLOGY IN PREGNANCY}

Changes in certain serum liver enzymes occur during normal pregnancy, and an understanding of these physiological changes is necessary for the management of liver diseases. ${ }^{3}$ The pregnant woman has physiological changes to support fetal growth and development. ${ }^{4}$ The levels of estrogens and progesterone increase during pregnancy. These sex hormones affect hepatic metabolic, synthetic, and excretory functions. ${ }^{5}$ Despite these diverse changes in function, no structural or morphological changes in hepatic tissue have been identified as a result of pregnancy. ${ }^{6}$

The plasma volume increases steadily from the sixth to the $36^{\text {th }}$ week of gestation by a total of about $50 \%$. The red cell volume also increases, but the increase is moderate (about $20 \%$ ) and delayed. Consequently, the total blood volume increases resulting in hemodilution. The hematocrit decreases by the $24^{\text {th }}$ week and becomes stable. Plasma and red cell volume decrease rapidly after delivery. ${ }^{7,8}$ This phenomenon of hemodilution should be considered during the interpretation of all laboratory tests during 
pregnancy. ${ }^{9}$ Hemodilution secondary to the increase in plasma volume decreases the serum protein concentrations and consequently leads to changes in values of liver enzymes during a normal pregnancy. Cardiac output increases (about 40\%) until the second trimester, then decreases, and normalizes near term. Absolute hepatic blood flow remains unchanged, but the percentage of cardiac output to the liver decreases. ${ }^{10}$

Typical measurements of liver function, including aspartate aminotransferase (AST), alanine aminotransferase (ALT), $\gamma$-glutamyl transferase (GGT), total bilirubin, and albumin, decrease slightly in concentration due to hemodilution, while alkaline phosphatase (AP) levels increase up to 4-fold relative to the non-gravid state. ${ }^{6,11}$ The increase in AP is due to the production of placental isoenzymes and is not a reflection of changes in maternal liver function. ${ }^{11}$ Due to these dilutional decreases, any increases in AST, ALT, or GGT in pregnancy may be ascribed to hepatic pathology. The $\alpha$-subunit of human chorionic gonadotropin (hCG) has a structure identical to that of luteinizing hormone (LH), follicle stimulating hormone (FSH), and, importantly, thyroid stimulating hormone (TSH). This similarity to TSH causes an increase in thyroid hormone $\left(T_{4}\right)$ production during pregnancy. However, accommodation for the increase in $T_{4}$ is achieved through equal increase in thyroid binding globin production by the liver, keeping levels of free $T_{4}$ relatively stable throughout pregnancy. ${ }^{6}$ Directly relevant to gastrointestinal bleeding in pregnancy, the liver increases synthesis of clotting factors V, VII, VIII, and fibrinogen. ${ }^{11}$ One might expect that these increases would result in a decreased propensity for bleeding. However, there is no measureable change in either prothrombin time (PT) or activated partial thromboplastin time (aPTT). ${ }^{11}$ Increases in leucine aminopeptidase, an enzyme with oxytocinase and vasopressinase function, increase urinary output through indirect action on the kidneys. ${ }^{6}$ In addition to these hepatic effects, the gallbladder loses contractility through the action of progesterone, which may result in intrahepatic cholestasis with associated pruritus despite the lack of increase in total bile acid concentration. ${ }^{6,11}$ Given the central catabolic and anabolic roles of the liver, it is evident that alterations in liver function can have far-reaching effects on the body.

\section{Pathophysiology}

The main maternal risk associated with pregnancy in cirrhosis is from portal hypertension. In a normal pregnancy, portal blood flow increases and drives more blood into the portosystemic collateral circulation. ${ }^{12,13}$ These changes exacerbate the pre-existing portal hypertension in cirrhosis and result in high complication risks: fetal demise in $42 \%$, maternal death in $3.4 \%$, and significant complications, including bleeding, encephalopathy, or ascites in $10 \%$ of cases. ${ }^{14}$ The risk of variceal bleeding is greatest in the second trimester and during delivery when increased pressure on the vena cava can increase portal vein pressure. Post-partum hemorrhage occurs in $7-10 \%$ of patients. Splenic artery aneurysm rupture can be a rare $(2.6 \%$ of patients) but fatal complication. Occasionally, hepatic encephalopathy, ascites, spontaneous bacterial peritonitis, and hepatic failure occur. ${ }^{15}$ In decompensated cirrhosis the anabolic function of the liver declines, resulting in a loss of essential clotting factors and thrombopoietin. Therefore, patients with severe liver disease are at a higher risk for life-threatening bleeding events either through pregnancy-related causes or through portal hypertension-related causes, especially due to the physiologic hemodilution of pregnancy. These coagulation abnormalities must be addressed before delivery - vaginal or Caesarean - to decrease the risk of maternal hemorrhage and must be monitored throughout pregnancy due to the risk of intrapartum gastrointestinal bleeding. However, since proteins $\mathrm{C}$ and $S$ and antithrombin are made by the liver and are anticoagulants, women with hepatic disease may suffer from excess thrombotic activity instead, depending on their unique disease state. ${ }^{16}$ Due to disruption of hepatic function, women suffering from decompensated cirrhosis have extreme difficultly becoming pregnant. Anovulatory cycles are more common, and the endocrine axes are disrupted, leading to infertility in most cases. ${ }^{17}$ However, pregnancy is still possible in the setting of severe liver disease, especially given the difficulty associated with hormonal contraception 
administration in patients with decreased hepatic function. ${ }^{18}$ Lee and Brady provide an excellent review of the effects of a wide range of liver diseases in pregnancy. ${ }^{19}$ They note that in cirrhosis with portal hypertension, vaginal birth should be avoided, complication and bleeding rates are high, and endoscopic and pharmacologic treatment is often indicated. We will discuss several cases, their choices for management, and their outcomes.

\section{REVIEW OF PUBLISHED OUTCOMES AND MANAGEMENT PRACTICES}

Rasheed et al present a prospective cohort study involving 129 pregnant cirrhotic women (study group), 647 pregnant non-cirrhotic women (control group 1), and 853 non-pregnant cirrhotic women (control group 2). ${ }^{20}$ They report an increase in complication rate from $8.2 \%$ to $31.8 \%$ comparing the study group to control group 1 with mortality rates at $7.8 \%$ and $0.2 \%$, respectively. Fetal mortality rates for cirrhotic women were $4.7 \%$ and for non-cirrhotic women were $0.8 \%$. The study group was more likely to suffer from preeclampsia $(12.4 \%$ vs $2.6 \%)$, thrombocytopenia ( $26.4 \%$ vs $0.5 \%)$, abruption $(10.1 \%$ vs $3.1 \%)$, or postpartum hemorrhage $(16.3 \%$ vs $5.1 \%)$. Additionally, $20.9 \%$ of study group participants received a blood transfusion, compared to $2.6 \%$ of control group 1 . Pregnancy also caused more rapid development of hepatic decompensation, with the study group decompensating in 33.4 weeks and control group 2 in 38.6 weeks. The study group developed gastrointestinal bleeding and encephalopathy, while control group 2 instead manifested decompensation as ascites and jaundice. Vaginal delivery increased the risk of severe bleeding during labor as compared to Cesarean delivery. Medical management was not discussed further.

A case series describing nine pregnancies occurring in seven patients was published by Aggarwal et al. ${ }^{17}$ Of these, 4 patients were diagnosed with cirrhosis and portal hypertension. Of those four, three developed esophageal varices. One then underwent sclerotherapy eight times for intractable varices and, after an episode of hematemesis, variceal ligation.
Another had sclerotherapy alone. The third received no treatment. In the case of the patient with portal hypertension but without varices, a lienorenal shunt had been placed. The only maternal death occurred in a patient suffering from autoimmune hepatitis, Cushing's syndrome, and membranous glomerulonephritis. In that case, the patient delivered at 29 weeks, experienced a postpartum hemorrhage and subsequent fulminant liver failure, dying eight days after delivery. Her only intrapartum complication was jaundice. The deceased patient also had chronic disease longer than any other patient in the study, 15 years compared to six years or less. These authors suggest hyperbilirubinemia is a marker of impending complications and decompensation and suggest aggressive prophylactic management involving sclerotherapy, ligation, and shunting to mitigate bleeding risk.

Esophageal bleeding secondary to variceal development in the setting of cirrhosis in pregnancy treated with a transjugular intrahepatic portosystemic shunt (TIPS) and variceal embolization was discussed by Lodato et al. ${ }^{21}$ The patient - at 14 weeks' gestation was anemic with thrombocytopenia, prolonged INR, active liver inflammation, and hypoalbuminemia. She experienced three episodes of severe esophageal bleeding and received atoxisclerol, as well as $\beta$-blockers, octreotide, and fresh frozen plasma and packed red blood cells during these bleeding events. After the third bleed, medical and supportive management was considered insufficient, and she underwent the TIPS procedure. The shunt failed to reduce the varices acceptably, and embolization was then performed. This resulted in a decrease in portal vein pressure to $26 \mathrm{mmHg}$. At 26 weeks she had premature rupture of membranes and bleeding. Premature labor is a significant risk (25\%) with concurrent cirrhosis, ${ }^{17}$ and these authors did not attribute it to the TIPS procedure, a rare event in pregnancy. ${ }^{21} \mathrm{~A}$ Cesarean section was performed, and the neonate (1035 grams) died 12 hours later from respiratory distress. They suggest TIPS as a last-choice effort in poorly-controlled frequent upper $\mathrm{Gl}$ bleeds.

A patient with a history of multiple substance abuse problems diagnosed with cirrhosis at 24 weeks was reported by Chaudhuri, Tan, and Biswas. ${ }^{22}$ 
Esophageal varices were banded at 30 weeks by endoscopy, and she was given propranolol for prophylaxis. She then progressed through vaginal labor at 38 weeks with no further episodes of hematemesis. Further esophageal bands were placed post-partum based on endoscopic findings. Despite her high risk of bleeding, this patient and her infant (2875 grams) were managed effectively with combination surgical and medication therapy.

Sobral et al discuss the case of a 35-year-old woman with a previously undiagnosed $\alpha 1$-antitrypsin deficiency. She had a successful term pregnancy before, but at 24 weeks she presented with hematemesis and melena. Liver inflammation and anemia were detected, but she became hemodynamically stable after packed red blood cell transfusion, and no ligation, sclerotherapy, or $\beta$-blocker administration was performed. She was followed closely with frequent appointments. The patient and her infant (3100 grams) remained stable after a Caesarean section at 39 weeks. Band ligation was performed in the post-partum period. This case suggests that with highly compliant patients and single episodes of bleeding, expectant management may produce good outcomes, though they acknowledge that the lack of consensus on whether prophylactic medical and/or surgical management are preferable to expectant management due to the lack of data on these management strategies.

The benefits of prophylactic treatment were described by Braga and Braga in their case report describing successful pregnancy in the setting of cirrhosis secondary to autoimmune hepatitis. ${ }^{23}$ This patient was continued on her chronic medications, including azathioprine (with folic acid supplementation), and was treated with propranolol, ranitidine, and prednisolone beginning at 7 weeks. She had no increase in liver enzymes except for a mildly elevated direct hyperbilirubinemia, a prothrombin time of 13.2 seconds, and she also had thrombocytopenia between $30 \mathrm{k}$ and $40 \mathrm{k}$ platelets. Mild upper gastrointestinal hypertension was noted during an endoscopy at 25 weeks but not surgically treated. She received a platelet transfusion before undergoing a Cesarean delivery secondary to fetal distress after spontaneous rupture of membranes at 37 weeks. She and her infant (2550 grams) progressed well in the post-partum period. As her disease was autoimmune in nature, the presence of a pregnancy may actually have improved her condition due to increased immune tolerance in the gravid state. However, she did have confirmed cirrhosis of the liver five years before becoming pregnant.

Chronic hepatitis B in a patient with a single successful pregnancy before the onset of liver cirrhosis and two unsuccessful pregnancies following was discussed by Kouakou et al. ${ }^{24}$ This patient became pregnant for a fourth time and had liver inflammation and a coagulopathy with thrombocytopenia and an increased prothrombin time. She was not evaluated by endoscopy. Massive ascites was present and later removed, and she was given a $\beta$-blocker for variceal prophylaxis and antibiotics to control the possibility of spontaneous bacterial peritonitis. Her bleeding risk was mitigated with fresh frozen plasma, packed red blood cells, and vitamin $\mathrm{K}$ infusions. At 35 weeks she went into spontaneous vaginal labor and gave birth vaginally. The infant (2150 grams) experienced neonatal distress syndrome.

\section{Management}

The management of GIB in cirrhotic pregnant patients is similar to non-pregnant patients with some exceptions. The management generally includes three components: preconception, during pregnancy, and in the acute setting. Preconception screening for varices is essential for optimal care. If no varices are identified or if no preconception screening is performed, endoscopic evaluation is recommended in the second trimester. If small varices have developed, beta blocker therapy should be started. Close fetal monitoring is important, as fetal bradycardia and intrauterine growth retardation may occur with beta blocker use. $^{12}$ Medium to large esophageal varices noted before conception, without history of hemorrhage, can be controlled with either beta blockers or endoscopic therapy. As the varices will likely increase during pregnancy, it seems reasonable to proceed with variceal band ligation. Esophagogastroduodenoscopy (EGD) in general appears to be safe during pregnancy but has a risk of fetal hypoxia from sedative drugs or positioning. ${ }^{25}$ Thrombocytopenia (platelet count of 
$<100$ cells/L), but not MELD score, can predict the presence of varices on screening endoscopies during the second trimester. ${ }^{14}$ It was reported that prophylactic band ligation for high-risk varices is a safe and effective method before and during pregnancy. ${ }^{13}$ Large varices with previous gastrointestinal bleeding should be ligated to obliteration, preferably before conception, and treated during pregnancy as required. For failure of endoscopic therapy or for large varices with preserved hepatic function, a shunt may be considered before pregnancy. In older literature, definitive surgical therapy for varices has been shown to improve fetal and maternal outcome. ${ }^{26,27}$ Acute variceal bleeding is managed endoscopically, as in the non-pregnant patient, with band ligation or sclerotherapy. Serial endoscopies for variceal band ligation to obliteration should be performed. Use of adjunctive medications during the bleeding episode is limited. Vasopressin is contraindicated due to risk of placental ischemia and potential teratogenicity. Octreotide use is controversial. Although it is an FDA category B drug, it causes splanchnic vasoconstriction and may precipitate placental ischemia and abruption, and its safety has not been well studied in pregnant patients. Transjugular intrahepatic portosystemic shunt is another option in failed endoscopic control of bleeding, but it will expose the fetus to considerable radiation and its use must be weighed against the mother's status. ${ }^{28}$ Delivery in these women is controversial but should be scheduled due to the risk of bleeding. ${ }^{29}$ The rise of the abdominal pressure due to Valsalva maneuvers during delivery can increase portal hypertension and cause bleeding from varices. Some authors recommend performing an elective Caesarean section or an assisted delivery with vacuum or forceps with epidural anesthesia. ${ }^{12,26}$ Our proposed management strategy for cirrhotic pregnant patients is described in the Figure.

Initial evaluation should consist of an esophagogastroduodenoscopy (EGD) to evaluate for varices or active bleeding. Preferably this should be done as early in the pregnancy as possible and whenever clinical suspicion suggests development or exacerbation of varices, weighed against risk of the procedure to the fetus. Depending on the size of the varices, different management approaches may be taken. Nonselective $\beta$-blockers should be used if indicated. If the patient presents for delivery of the fetus and her risk is unknown or is determined to be high, a Caesarean section or assisted delivery is indicated to prevent the development of high intra-abdominal pressures from the Valsalva maneuver.

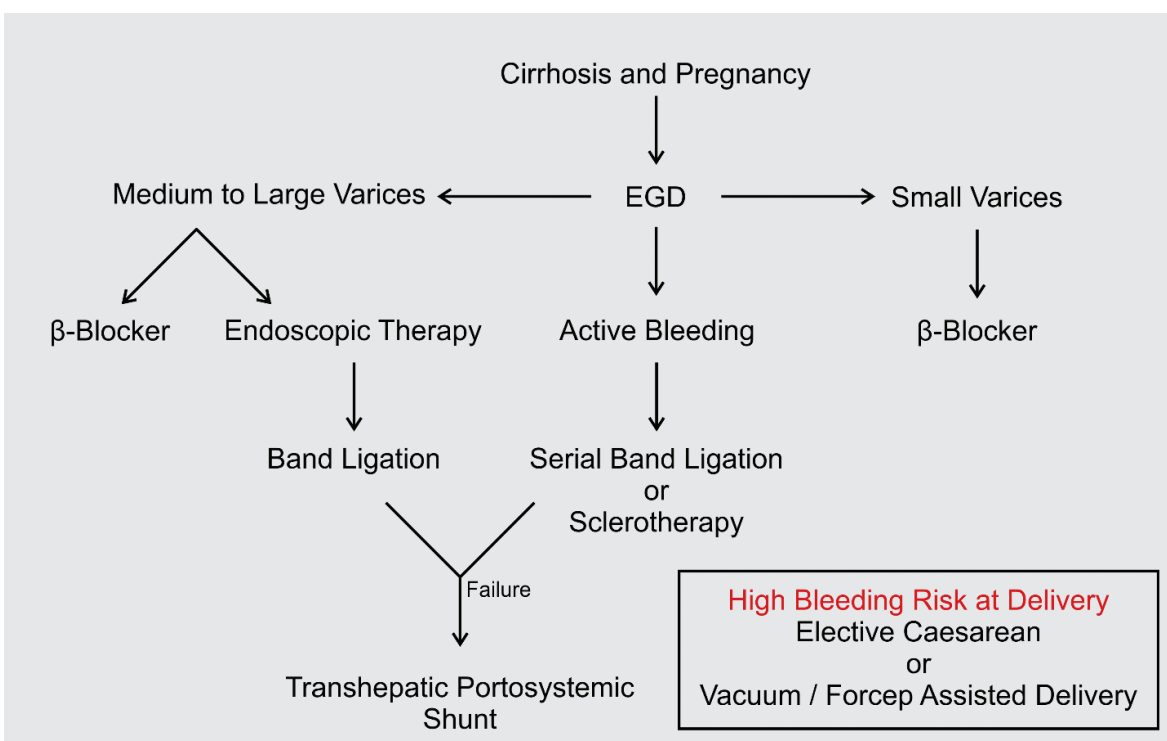

Figure. Management strategy for the pregnant cirrhotic patient. 


\section{ConcLusion}

Although the current management of liver disease (GIB) and associated complications is similar in pregnant and non-pregnant women, there are significant physiological differences in natural progression and treatment response that need better understanding to optimize future management of GIB in pregnancy. Successful pregnancy is possible in inactive, compensated cirrhosis, but it is still carries high risk and demands careful counselling about maternal and fetal risk. The pathophysiology of severe liver disease results in difficulty in becoming pregnant, a statistic highlighted by the case reports we have described here. This difficulty results in relatively low numbers of pregnant women with decompensated liver disease, one limitation of this case series and review. Additionally, within this small patient population, treatment protocols appear to be relatively diverse and highly dependent upon the resources available at the admitting hospital and the other comorbid conditions of the patient. However, esophageal banding or sclerotherapy versus medical management appear to be the two mainstays of treatment. A complete meta-analysis would be beneficial in determining whether surgical or medical intervention is better for this unique patient population. Despite these differences, all case reports underscore that active management of varices is essential before and during pregnancy. Contraception must be discussed with women of child-bearing age to avoid unwanted and potentially dangerous pregnancies, especially in this difficult-to-manage population.

Article citation: Shredi A, Elberson B. Gastrointestinal bleeding in pregnant patients with cirrhosis: pathophysiology, review of outcomes, and management. The Southwest Respiratory and Critical Care Chronicles 2018;6(25):19-25

From: The Department of Internal Medicine at Texas Tech University Health Sciences Center in Lubbock, Texas

Submitted: $4 / 8 / 2018$

Accepted: 6/15/2018

Reviewers: Ariwan Rakvit MD, Edward Yeomans MD

Conflicts of interest: None

This work is licensed under a Creative Commons Attribution-ShareAlike 4.0 International License

\section{REFERENCES}

1. Tan J, Surti B, Saab S. Pregnancy and cirrhosis. Liver Transpl 2008;14:1081-91

2. Subhan A, Abid S, Jafri W. Successful outcome of a pregnancy in a woman with advanced cirrhosis due to hepatitis B surface antigenemia, delta super-infection and hepatitis C co-infection: a case report. J Med Case Rep 2007;1:96.

3. Bacq Y. Liver and pregnancy. Pathol Biol (Paris) 1999;47: 958-65.

4. Blackburn ST, Loper DL. Maternal, Fetal, and Neonatal Physiology: A Clinical Perspective. Philadelphia, PA: Saunders; 1992.

5. Van Thiel DH, Gavaler JS. Pregnancy-associated sex steroids and their effects on the liver. Semin Liver Dis 1987;7:1-7.

6. Cunningham FGL, K. J., Bloom SL, Spong CY, et al. Williams Obstetrics. New York: McGraw-Hill Education; 2014.

7. Lund CJ, Donovan JC. Blood volume during pregnancy. Significance of plasma and red cell volumes. Am J Obstet Gynecol 1967;98:394-403.

8. Peck TM, Arias F. Hematologic changes associated with pregnancy. Clin Obstet Gynecol 1979;22:785-98.

9. Goodlin RC. Serum albumin reserve for bilirubin binding during pregnancy in healthy women. Obstet Gynecol 1989;73:1054.

10. Munnell EW, Taylor HC. Liver blood flow in pregnancyHepatic vein catheterization. J Clin Invest 1947;26:952-6.

11. Mufti AR, Reau N. Liver disease in pregnancy. Clin Liver Dis 2012;16:247-69.

12. Russell MA, Craigo SD. Cirrhosis and portal hypertension in pregnancy. Semin Perinatol 1998;22:156-65.

13. Sandhu BS, Sanyal AJ. Pregnancy and liver disease. Gastroenterol Clin North Am 2003;32:407-36, ix.

14. Westbrook RH, Yeoman AD, O'Grady JG, et al. Model for endstage liver disease score predicts outcome in cirrhotic patients during pregnancy. Clin Gastroenterol Hepatol 2011;9:694-9.

15. Allen AM, Hay JE. Review article: the management of cirrhosis in women. Aliment Pharmacol Ther 2014;40:1146-54.

16. von Köckritz L, De Gottardi A, Trebicka J, et al. Portal vein thrombosis in patients with cirrhosis. Gastroenterol Rep (Oxf) 2017:148-56.

17. Aggarwal N, Sawnhey H, Suril V, et al. Pregnancy and cirrhosis of the liver. Aust N Z J Obstet Gynaecol 1999;39:503-6.

18. Jabiry-Zieniewicz Z, Dabrowski FA, Suchonska B, et al. Pregnancy and delivery in women with esophageal varices due to hepatic vein thrombosis. J Matern Fetal Neonatal Med 2015;28:177-81.

19. Lee NM, Brady CW. Liver disease in pregnancy. World J Gastroenterol 2009;15:897-906.

20. Rasheed SM, Abdel Monem AM, Abd Ellah AH, et al. Prognosis and determinants of pregnancy outcome among 
patients with post-hepatitis liver cirrhosis. Int J Gynaecol Obstet 2013:121:247-51.

21. Lodato F, Cappelli A, Montagnani M, et al. Transjugular intrahepatic portosystemic shunt: a case report of rescue management of unrestrainable variceal bleeding in a pregnant woman. Dig Liver Dis 2008;40:387-90.

22. Chaudhuri K, Tan EK, Biswas A. Successful pregnancy in a woman with liver cirrhosis complicated by recurrent variceal bleeding. J Obstet Gynaecol 2012;32:490-1.

23. Braga A, Braga J. Successful pregnancy with autoimmune cirrhosis. BMJ Case Rep 2016;2016.

24. Kouakou F, Loue V, Adjoby R, et al. Decompensated cirrhosis and pregnancy: a case report. Clin Exp Obstet Gynecol 2012;39:252-4.
25. O'Mahony S. Endoscopy in pregnancy. Best Pract Res Clin Gastroenterol 2007;21:893-9.

26. Schreyer P, Caspi E, El-Hindi JM, et al. Cirrhosis-pregnancy and delivery: a review. Obstet Gynecol Surv 1982;37:304-12.

27. Cheng YS. Pregnancy in liver cirrhosis and/or portal hypertension. Am J Obstet Gynecol 1977;128:812-22.

28. Savage C, Patel J, Lepe MR, et al. Transjugular intrahepatic portosystemic shunt creation for recurrent gastrointestinal bleeding during pregnancy. J Vasc Interv Radiol 2007; 18:902-4.

29. Heriot JA, Steven CM, Sattin RS. Elective forceps delivery and extradural anaesthesia in a primigravida with portal hypertension and oesophageal varices. Br J Anaesth 1996;76: 325-7. 